\title{
Treatment gains from the "sandwich method" of abiraterone acetate for men with metastatic prostate cancer: a case report and sharing of our experience
}

\author{
Hui Wang ${ }^{1}$, Xin $^{1}{ }^{1}$, Ping Zhang ${ }^{2}$, Shixiong Liu ${ }^{1}$, Qiang Xie ${ }^{3}$, Shankun Zhao ${ }^{1 \wedge}$ \\ ${ }^{1}$ Department of Urology, Taizhou Central Hospital (Taizhou University Hospital), Taizhou 318000, China; ${ }^{2}$ International Eye Hospital of Taizhou, \\ Taizhou 318000, China; ${ }^{3}$ Department of Reproduction, Southern Medical University Affiliate Dongguan People's Hospital, Dongguan 523059, \\ China \\ Correspondence to: Shankun Zhao, MD. Department of Urology, Taizhou Central Hospital (Taizhou University Hospital), Taizhou 318000, China. \\ Email: zskzp567@163.com.
}

\begin{abstract}
To date, how to select an appropriate strategy to treat advanced prostate cancer (PCa) patients with tumor progression after abiraterone acetate (AA) intervention is still confusing. Here, we conducted a case report and review of the literatures focused on this issue. A 63 -year-old man presented initially with dysuria. Multiple pelvic bone metastases but no other lesions were detected. He was subsequently diagnosed with PCa after biopsies, the initial clinical staging was cT2cNxM1. This patient was treated with two periods of AA (first treatment: 16-month; secondary treatment: 19-month) combined with other therapeutic regimens [i.e., goserelin acetate, bicalutamide, docetaxel, and radical prostatectomy (RP), etc.], followed by a significant biochemical response during over 5 years of follow-up. In the present case report with 63 months of the treatment course, we found that patient with metastatic PCa who early received AA as the initial regimen could gain benefit from the retreatment of AA: minor progression and gain long-time survival. This "sandwich method" with AA administered initially, withdrawal, and sequentially following Bicalutamide, Docetaxel, RP, and retreatment of AA may prolong the response time of AA retreatment and delay the progression of metastatic $\mathrm{PCa}$, which reminds clinicians should pay attention to this phenomenon and explore the underlying mechanisms.
\end{abstract}

Keywords: Abiraterone acetate (AA); prostate cancer (PCa); metastasis; sandwich method; case report

Submitted Dec 10, 2019. Accepted for publication Apr 24, 2020.

doi: $10.21037 /$ tau-19-851

View this article at: http://dx.doi.org/10.21037/tau-19-851

\section{Introduction}

Prostate cancer (PCa) is the most commonly diagnosed cancer among men in over 100 countries (1). Based on the Cancer Statistics in 2015, it was reported that the incidence and mortality rates of PCa increased annually in China (2). At present, metastasis remains the primary cause for most PCa deaths (3). There was $12 \%$ and $4 \%$ of the newly diagnosed PCa patients were identified to have invasive lymph node metastases and distant metastases in the US (4). However, it was reported that over half of the PCa patients in China have developed distant metastases at the time of the initial diagnosis of $\mathrm{PCa}$ (5). Metastatic PCa patients mostly received androgen deprivation therapy (ADT), and the 5-year overall survival of those patients was only $30 \%$. Moreover, a majority of PCa patients may

\footnotetext{
^, ORCID: 0000-0002-0305-3912
} 
experience progression to castration-resistant PCa (CRPC) after a median of 13-33 months of ADT (6). The median overall survival of metastatic CRPC (mCRPC) was only 12.3 months (7). Presently, metastasis remains a primary challenge in treating $\mathrm{PCa}$.

Abiraterone acetate (AA), a selective inhibitor of androgen biosynthesis in the testes, adrenal glands, as well as prostate cancer cells resulting in virtually undetectable serum and intratumoral androgens, is approved to treat both metastatic hormone-sensitive prostate cancer (mHSPC) and $\mathrm{mCRPC}$ either before or after docetaxel as combined with prednisone or prednisolone (8). AA is considered to be the first-line option for $\mathrm{mCRPC}$ in the National Comprehensive Cancer Network (NCCN) guidelines. Though effectively, we should know that almost all of PCa patients existed tumor progression after AA therapy due to the resistance. Thus, it is not acceptable to employ AA as the heal-over-time therapy. To date, how to select the appropriate strategy to treat those PCa patients with tumor progression after AA intervention is still confusing, especially in China where enzalutamide is not available yet. Most PCa patients only have one course of AA treatment in China because the time to the Chinese market is relatively short.

In this study, we reported a case of $\mathrm{mCRPC}$ in a patient treated with two periods of AA (first treatment: 16-month; secondary treatment: 19-month) combined with other therapeutic regimens [i.e., goserelin acetate, bicalutamide, docetaxel, and radical prostatectomy (RP), etc.], followed by a significant biochemical response during over 5 years of follow-up. The purpose of this study is to critically analyze the prognostic value of secondary AA therapy in mCRPC patients and conduct a systematic review of the relevant studies. We present the following case in accordance with the CARE reporting checklist (available at http://dx.doi. org/10.21037/tau-19-851).

\section{Case presentation}

\section{General information}

This study was approved by the Human Study Ethics Committees of the Taizhou Central Hospital (Taizhou University Hospital). The patient has signature informed consent. Human specimens were handled and made anonymous in adherence to ethical and legal standards.

This patient was a 63 -year-old man $(168 \mathrm{~cm}$ in height, weighs $69 \mathrm{~kg}$, 0 score for WHO performance status) without a past medical history of hypertension, diabetes mellitus, hyperlipidemia, coronary heart disease, trauma, or surgeries when first examined. This patient did not have a positive family history for cancers and no genetic susceptibility to cancers. Besides, no abnormalities of psychosocial characteristics were detected.

\section{Initial evaluation}

The patient presented to the urology department in another hospital with complaints of dysuria in January 2014. The level of prostate-specific antigen (PSA) was $258.0 \mathrm{ng} / \mathrm{mL}$ at the initial examination. The physical examinations were listed as follows, Eastern Cooperative Oncology Group (ECOG): 0 score; temperature: $37.0{ }^{\circ} \mathrm{C}$; pulse: 76 beats per minute; respiration: 20 breaths per minute; blood pressure: 120/80 mmHg. The patient was conscious, and the skinmucous membrane, thorax, respiration, and heart rhythm were normal. The abdomen was soft and no tenderness. No enlarged superficial lymph nodes, liver, and spleen were found. No edema of both lower limbs. Digital rectal examination showed a $\mathrm{II}^{\circ}$ to $\mathrm{III}^{\circ}$ enlarged prostate and there was no adhesion with the rectum. Magnetic resonance imaging (MRI) indicated abnormal signals in the peripheral zone of the right lobe of the prostate, considered as $\mathrm{PCa}$; benign prostatic hyperplasia; multiple abnormal signals in the right side of the femoral head and femoral neck, left acetabulum, and the sacrum, considered as metastatic tumors. The pathology reports of transrectal prostate biopsies indicated that positive cancer tissues were found in $4 / 5$ of needles in the left lobe of the prostate (including one needle in the apex of the prostate) and 4/5 of needles in the right lobe of the prostate (including one needle in the apex of the prostate). The patient's Gleason score was $4+4=8 / 10$. The results of emission computed tomography showed that multiple metastatic lesions were located in the pelvic organs but not observed in other sites (Figure S1A), which could be categorized as oligometastatic PCa. Thus, the initial clinical staging was c $\mathrm{T} 2 \mathrm{cNxM} 1$.

\section{Treatment regimens}

In the present case, this patient showed high intervention adherence and well tolerability. The only reason for changing the treatment strategy is the progression of the disease, that is continuously elevation of the PSA level, the occurrence of new clinical adverse events, and radiographic progression-the presence of two or more. 
Since the initial treatment till now, this patient did not complaint with any ostealgia, pathological fracture, anemia, depression, and cardiovascular events, etc. Therefore, in addition to constantly increasing PSA, any radiographic progression findings (i.e., MRI and CT scan of the pelvis, bone scintigraphy) were considered to change the treatment regimen.

\section{Initial systemic treatment}

This patient began to receive endocrine therapies in February 2014. Goserelin acetate (Zoladex, AstraZeneca UK Limited) was served as the base ADT therapy up to the present. The usage was subcutaneous goserelin acetate 3.6 $\mathrm{mg}$ monthly. The serum testosterone levels were confirmed to be $<50 \mathrm{ng} / \mathrm{dL}$ till now and the lowest point was recorded at $0.0 \mathrm{ng} / \mathrm{dL}$. Simultaneously, the patient was treated with 1,000 mg AA (Zytiga, Xian Janssen Pharmaceutical Ltd.) daily in combination with $5 \mathrm{mg} / 12 \mathrm{~h}$ prednisolone until June 2015. The lowest PSA level of this patient at this cycle was $0.37 \mathrm{ng} / \mathrm{mL}$ in November 2014. Since then, the PSA level was gradually elevated and was up to $9.31 \mathrm{ng} / \mathrm{mL}$ in June 2015. And the bone scintigraphy examination in May 2015 revealed that pelvic bones metastases were more aggressive than that in January 2015 (Figure S1B). Due to the serum PSA level increased and the metastases advanced, consequently, the first course of AA treatment was withdrawn. In the first AA regimen, the patient completed a total of 16 months of treatment. Of note, AA (Zytiga) was received approval of a new drug application for mCRPC in China in January 2016. In this case, the patient could be treated by AA early in February 2014 due to the reason that he participated in the Chinese Clinical Trial of AA.

\section{Androgen blockade treatment}

Then, the patient switched to bicalutamide (Casodex, Xian Janssen Pharmaceutical Ltd.) $50 \mathrm{mg}$ once daily in June 2015. However, this attempt has resulted in a poor response. The PSA presented no downward tendency but rose continually up to $160.0 \mathrm{ng} / \mathrm{mL}$ in June 2016. Of note, the initial treatment of AA and the bicalutamide therapy were conducted in other hospitals, so we could not get the entire radiographic data of this patient and thus failed to fully explain the rationale of changes in treatment.

\section{Chemotherapy}

In June 2016, this patient was referred to our hospital for chemotherapy which was recommended by a urologist from another hospital. We noted that the PSA level of this patient continually increased. Moreover, the patient complained with a radiographic progression but failed to provide the corresponding imaging data. Considering the castration-resistant disease, the first systemic chemotherapy with docetaxel was performed. Before chemotherapy, we had performed a physical examination for this patient. The consciousness, blood pressure, heart rate, respiration, and temperature were unremarkable. Heart and lungs examinations were normal. The abdomen was soft and no abdominal mass was detected. No bilateral inguinal lymph nodes enlargement was found. Digital rectal examination revealed an irregularly enlarged $\left(\mathrm{II}^{\circ}\right)$, stony, nodular (a $1.5 \times 1.5 \mathrm{~cm}^{2}$ hard, immobile, and painless nodule arising from the right lobe) prostate. Administration regimens were intravenous docetaxel (Hengrui Pharmaceutical) $75 \mathrm{mg} / \mathrm{m}^{2}$ triweekly and PO prednisone $5 \mathrm{mg} / 12 \mathrm{~h}$. He completed 10 cycles of treatment (June 2016 to January 2017). The patient's PSA level analytical nadir reached $1.16 \mathrm{ng} / \mathrm{mL}$ after chemotherapy was completed. Bone scintigraphy (December 2016) indicated that the lesions in the pelvic region were alleviative than that in May 2015, and no other new metastases were detected (Figure 1). In the following 3 months afterward, this patient has only received goserelin acetate treatment; his PSA gradually rose to $6.03 \mathrm{ng} / \mathrm{mL}$.

\section{RP}

In April 2017, the patient has received a successful RP under laparoscope, and his urination function was completely recovered in 6 months after the operation.

\section{Preoperative assessment}

Preoperatively, the PSA level of this patient was $6.03 \mathrm{ng} / \mathrm{mL}$. His prostate was $\mathrm{II}^{\circ}$ enlarged, hard, fixation, without tenderness. A $1.5 \times 1.5 \mathrm{~cm}^{2}$ prostatic tubercle in the right lobe was detected by DRE. The transrectal ultrasound indicated that his prostate gland was $41 \times 35 \times 30 \mathrm{~mm}^{3}$ in size, and inhomogeneous internal echoes inside (Figure $2 A$ ). Results from MRI showed that some abnormal signals in the peripheral zone of the right lobe of the prostate; T2W showed low signal intensity, and nodules broke through the capsule, and DWI presenting high signal intensity; There was no abnormal metastatic signal in pelvic lymph nodes and other sites (Figure 2B,C). Bone scanning indicated that the lesions in the pelvic region were more advanced than those in December 2016, but no other metastatic lesions were found (Figure 3). At this time, due to this patient was diagnosed with "oligometastatic $\mathrm{PCa}$ " and the next treatment regimen is still controversial yet, we 


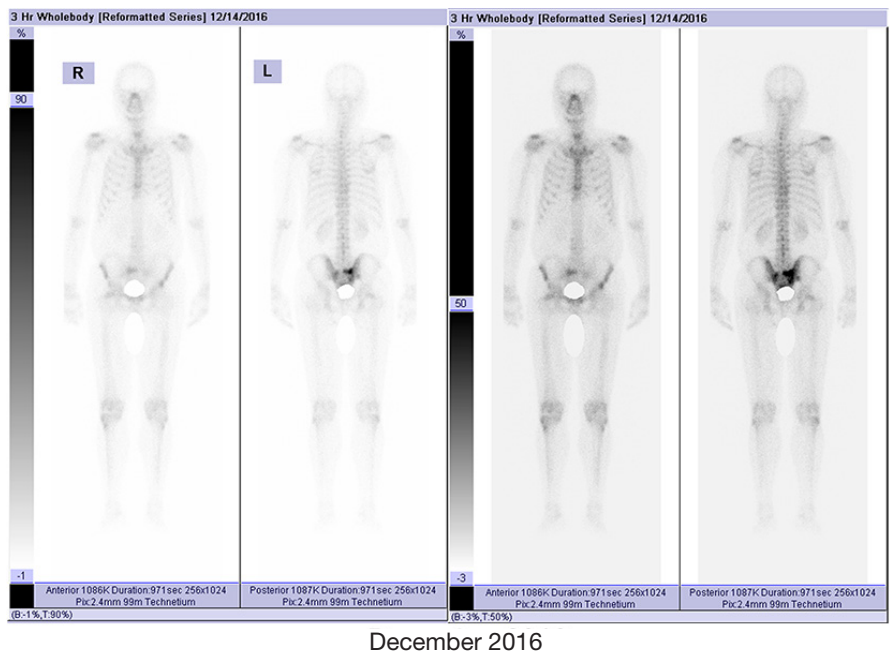

Figure 1 Emission computed tomography evaluation at the end of chemotherapy (docetaxel) in December 2016.
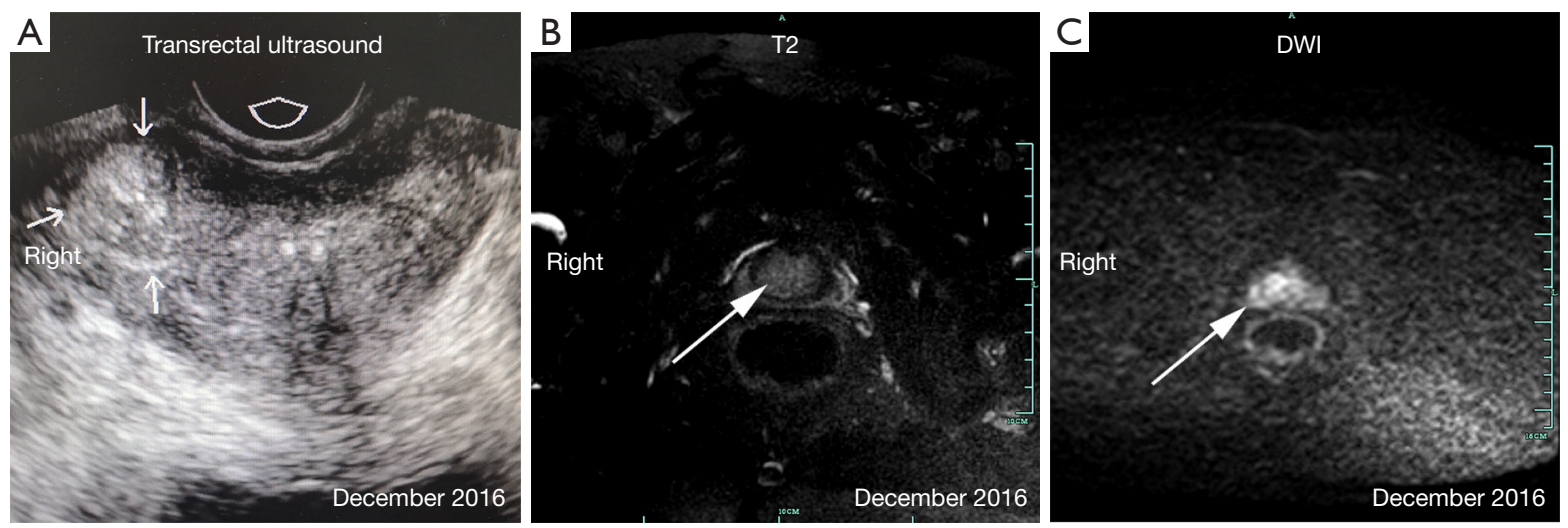

Figure 2 Preoperative findings from transrectal ultrasound and magnetic resonance imaging (MRI). (A) Transrectal ultrasound indicated that inhomogenous internal echoes inside the prostate; (B,C) preoperatively, T2W and DWI of MRI revealed the abnormal signals in the prostate. The arrows indicate the tumor of the prostate.

recommended a radical excision of the carcinomatous prostate under patients' informed consent.

\section{Postoperative assessment}

Postoperatively, the pathological report showed that there was one lesion in the middle of the right lobe with a diameter of $1.6 \mathrm{~cm}$ and one lesion in the apex of the left lobe with a diameter of $1.5 \mathrm{~cm}$, both of which involved extra-capsular adipose tissue of the prostate. The bilateral seminal vesicle, vas deferens, urethral margin, and bilateral pelvic lymph nodes (a total of 13 nodes) were negative for carcinoma. The Gleason score was 4+5=9/10 (Figure 4). The PSA continued to rise to $18.25 \mathrm{ng} / \mathrm{mL}$ in six weeks after the operation. At four months postoperatively (August 2017), the physical examination showed that the vital signs of this patient were normal, no abdominal mass was found, and bilateral inguinal lymph nodes were negative. No prostate and rectal mass were palpable on digital rectal examination. MRI showed that the prostate and its appendages were resected after RP, and the surrounding structures were clear. There was no abnormal signal and no enhancement after examination of the MRI enhancement. No abnormality was found in the size, shape, and signal of the bladder. No enlarged lymphoid nodules were found near the iliac vessels on both sides, and no effusion was detected in the pelvic area (Figure 5). Bone scintigraphy in August 2017 showed 


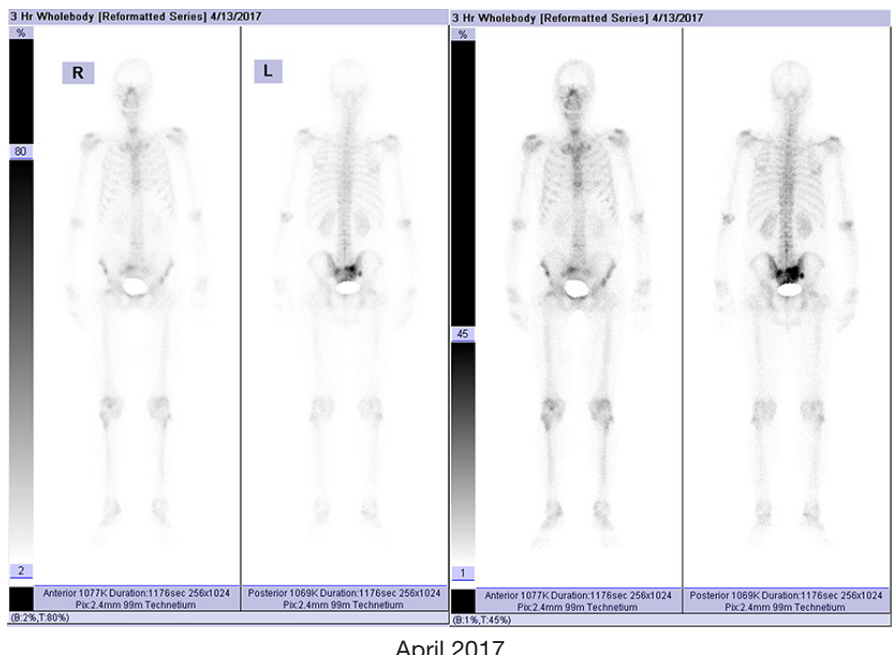

Figure 3 Preoperative bone scintigraphy assessment in April 2017.
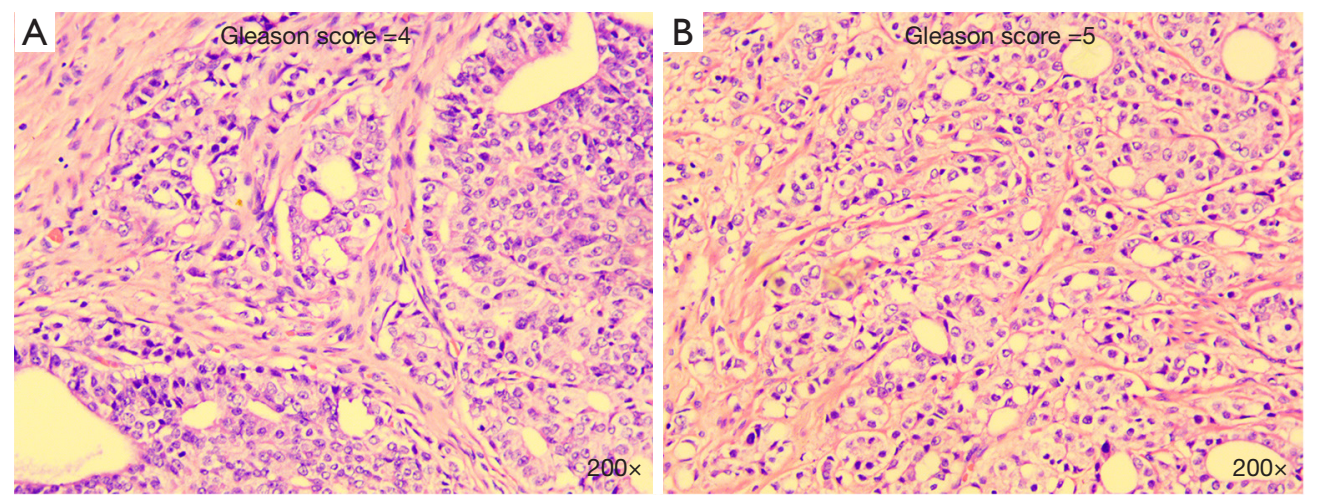

Figure 4 Representative radical prostatectomy sections of cancerous by HE staining at 200×. (A) Gleason score =4; (B) Gleason score =5 .
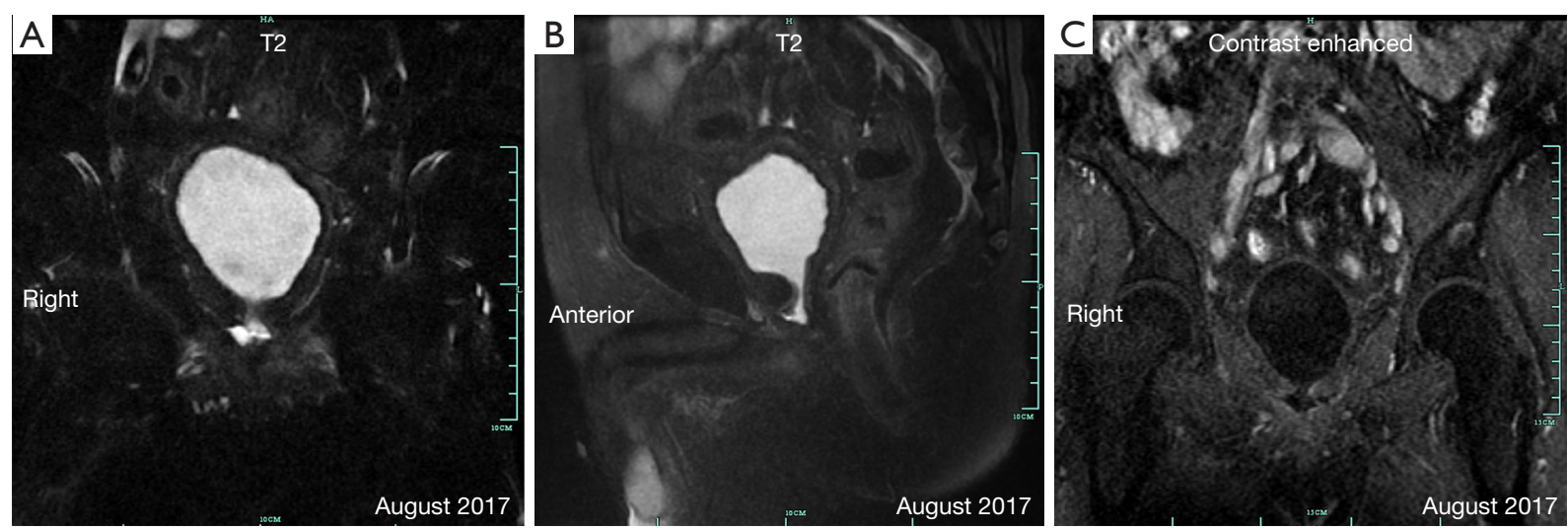

Figure 5 Postoperative findings from transrectal ultrasound and MRI. (A,B,C) Postoperatively, MRI and contrast enhanced-MRI showed the prostate and its appendages were resected and no enlarged lymphoid nodules were found. 

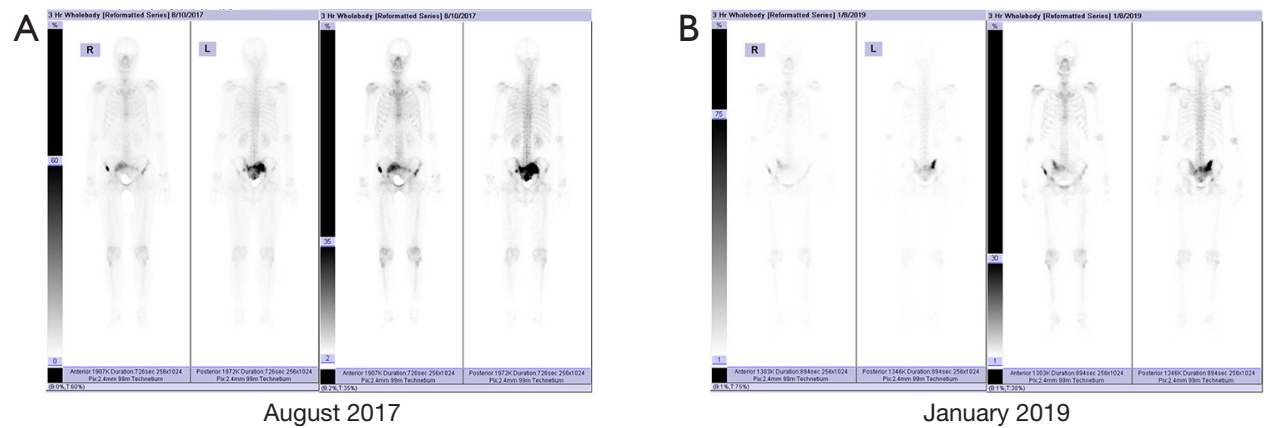

Figure 6 Other bone scintigraphy findings. (A) Postoperative evaluation at four months after radical prostatectomy (August 2017); (B) assessment at the end of retreatment of AA in January 2019. AA, abiraterone acetate.

that the lesions in the pelvic region were worsened than that of April 2017, but no metastases were detected other than the pelvis (Figure 6A). Based on these signs, the present clinical staging was $\mathrm{pT} 3 \mathrm{aN} 0 \mathrm{M} 1$.

\section{Retreatment of AA}

In September 2017, the PSA level of this patient continued to rose to $336 \mathrm{ng} / \mathrm{mL}$. Combined with lesions in the pelvic region were worsened than that of preoperative situation, repeatedly, he had received a secondary AA treatment, and the dosing regimen was the same as the first administration $(1,000 \mathrm{mg}$ AA daily in combination with $5 \mathrm{mg} / 12 \mathrm{~h}$ prednisolone). The level of PSA at this treatment cycle decreased to the lowest point of $0.85 \mathrm{ng} / \mathrm{mL}$ in June 2018, but subsequently continued rose to $76.22 \mathrm{ng} / \mathrm{mL}$ till April 2019. Reexamination of bone scintigraphy (January 2019) indicated that the range of pelvic lesions was smaller than that in August 2017, and no new metastasis was found in the other regions (Figure 6B). Though the pelvic metastases were alleviated, the secondary course of AA treatment was withdrawn at this time due to the serum PSA level gradually increased. And fortunately, this patient met the inclusion criteria of a new pharmaceutical clinical trial in China and thus entered that multicenter double-blind randomized controlled clinical trials, the therapeutic outcomes were not available now. In the secondary AA regimen, this patient completed a total of 19 months of treatment.

\section{Evaluation of therapeutic efficacy}

The evaluation methods of therapeutic efficacy in PCa included PSA progression, radiographic progression, and clinical symptoms progression. Two of the three advances were evaluated as tumor progression.

\section{Results}

From the initial systemic treatment till now, there have been no clinical events presenting on this patient, such as dysuria and bone pain, etc. From January 2014 to April 2019, the overall variations trend of the PSA level could be summarized as three "troughs" and two "peaks". The three "troughs" were the initial treatment of AA, chemotherapy, and retreatment of AA, and the two "peaks" were the time points that replaced with bicalutamide after the first treatment of AA and post RP (Figure 7).

During the whole course of management, this patient displayed a high intervention adherence, he was willing to cooperate with all the recommended treatments and was able to return to the hospital on time to accept the necessary laboratorial and radiographic examinations. In addition, this patient also showed well tolerability when ongoing different therapeutic regimens. The patient did not develop any clinical adverse events at all stages of treatment, including abnormal vital signs, diarrhea, vomiting, anorexia, depression, rash, $\mathrm{III}^{\circ}$ of myelosuppression, abnormal liver and renal function, and hyperglycemia, etc.

\section{Discussion}

In the newly diagnosed PCa patients in the United States, it was reported that there were $81 \%, 12 \%$ and $4 \%$ of the patients were diagnosed to clinical localized $\mathrm{PCa}$, having invasive lymph node metastases, and existing distant metastases, respectively (4). However, data from multicenter 


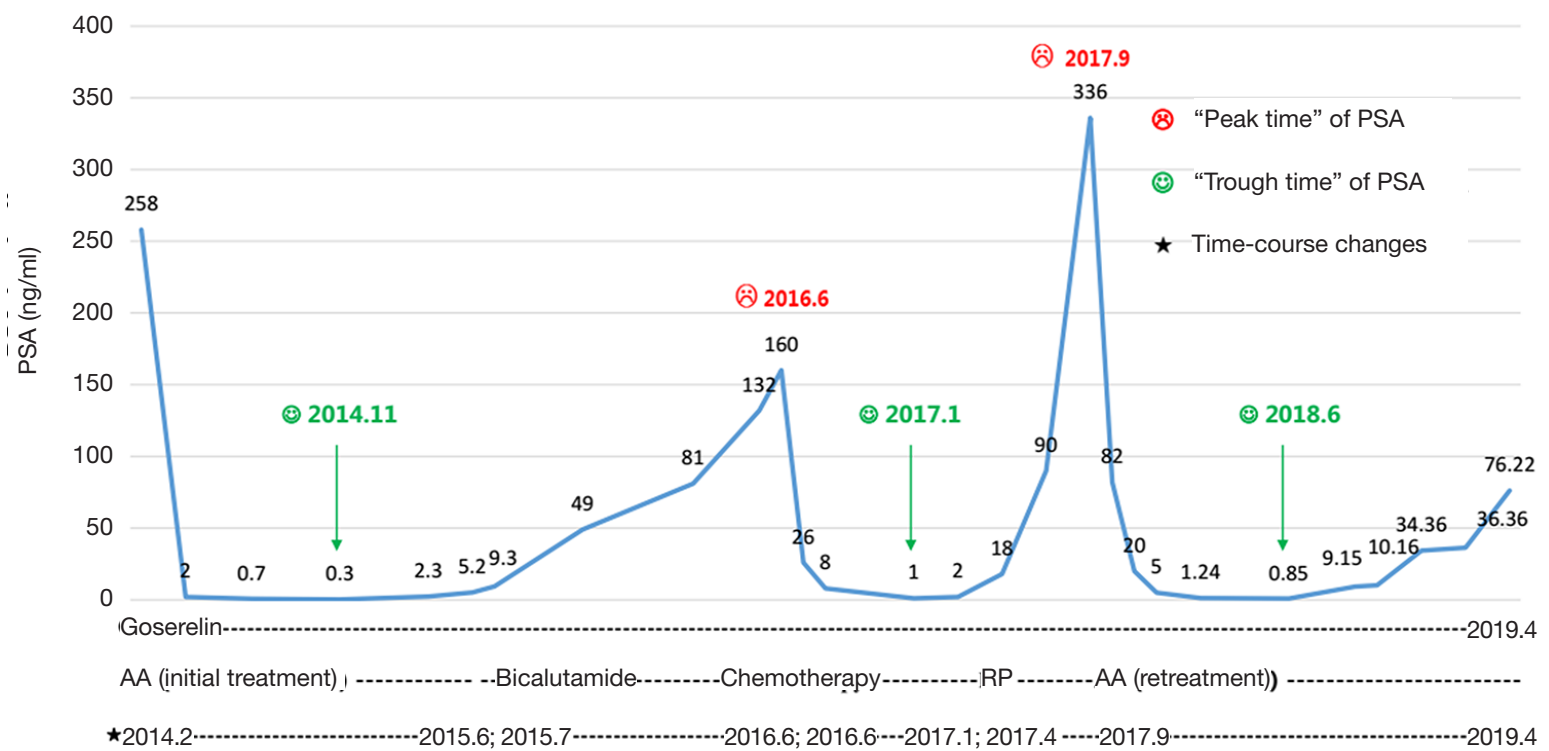

Figure 7 Time-course changes of serum PSA levels and therapeutic agents. The three "troughs" were recorded in the initial treatment of AA, chemotherapy, and retreatment of AA, and the two "peaks" were the time points that replaced with bicalutamide after the first treatment of AA and postoperative radical prostatectomy. PSA, prostate-specific antigen; AA, abiraterone acetate.

studies in China showed that more than $50 \%$ of PCa patients have developed distant metastases, such as bone and viscera, at the time of initial diagnosis, resulting in the overall prognosis of $\mathrm{PCa}$ patients in China is much worse than that in western countries (5). For those metastatic PCa patients who mainly received $\mathrm{ADT}$ as the palliative treatment, their 5 -year overall survival was only $30 \%$. For $\mathrm{mPCa}$, it needs a comprehensive intervention that combined a variety of treating methods, including medications or surgical ADT, anti-androgen therapy, RP, internal radiotherapy, external radiotherapy, chemotherapy, and immunotherapy, etc. Once the disease shifts to the mCRPC stage, the original anti-androgen therapy is no longer effective, and new therapeutic medications need to be replaced continuously. Therefore, the primary goal of mCRPC treatment is to prolong the validity of antiandrogenic drugs. Presently, only a few kinds of first-line anti-androgen medications attained the official certification in China, mainly bicalutamide, AA, and apalutamide (just approved in September 2019). However, there are other treatment options in foreign countries, such as enzalutamide, radium Ra 223 dichloride, cabazitaxel, and sipelucel-T, but these options are not yet available in China.

In 1994, Barrie et al. first described several novel steroidal inhibitors of cytochrome P450 alpha (17 alpha-hydroxylase/ C17-20 lyase) which could dramatically reduce plasma testosterone (9). One of these agents, AA, is a selective, potent oral micro-molecule inhibitor of $17 \alpha$-hydroxylase/ C17,20-lyase (CYP17), which can significantly interfere with the biosynthesis of androgens in the adrenal glands, testes, and PCa cells and thereby remarkably reduce the androgen production (10). Major breakthroughs have been made in the treatment of mHSPC in the last 5 years. In the past, mHSPC was treated with conventional endocrine therapy until 2015. In 2015, the CHAARTED study showed that the early usage of ADT plus chemotherapy (docetaxel) might be beneficial to patients with mHSPC (11). Of note, the replacement and even withdrawal of the anti-androgen medications are only to delay the timepoint of patients come into the chemotherapy or prolong the treatment cycle of chemotherapeutic drugs. Subsequently, the 2016 STAMPEDE study (12) and the 2017 LATTITUDE study (13) suggested that MHSPC patients could benefit from early AA. The 2018 edition EAU (14) and AUA (15) guidelines recommended both AA and docetaxel as the first-line regimens for mHSPC treatment. In a more recent study, Hoyle AP et al. demonstrated that patients gain treatment benefit from ADT plus AAP not only in "high-risk" metastatic hormone-naïve $\mathrm{PCa}$ (mHNPC) but also in "lowrisk" mHNPC, which indicates that AA is suitable in all men with metastatic disease starting hormone therapy for the first time (16). Note that the COU-AA-302 stratified study (17) showed that the median survival benefit of mCRPC patients 
received AA plus prednisone (AAP) treatment and without a chemotherapy history was 11.8 months when compared with prednisone alone (53.6 vs. 41.8 months). On the other hand, the COU-AA-301 study (18) indicated that APP had a median survival benefit of 4.6 months (15.8 vs. 11.2 months) in $\mathrm{mCRPC}$ patients with a history of chemotherapy. These data revealed that those mCRPC patients who early preferred AA would be more beneficial than docetaxel administration. One explanation for these observations is that there is cross-resistance between new antiandrogenic medications (i.e., AA) and chemotherapeutic drugs (i.e., taxanes) (19). Notably, the mechanism for traditional second-line antiandrogen medications (i.e., bicalutamide, flutamide, and ketoconazole) to reduce the androgen production is to target the androgen signaling pathway, taking ketoconazole as an example: its structure or functioning is highly similar to AA, thus there must be existed a cross-resistance between the two medications. Therefore, theoretically, mCRPC patients firstly received traditional second-line anti-androgen drugs that may affect the efficacy of the new generational antiandrogen medications. On the other hand, these traditional medications can only inhibit the tumor progression for a limited period, so the patients are unable to live for a better and longer survival by these drugs $(20,21)$. Based on the above evidence, several observations in the present study could be explained rationally. For example, it was ineffective to apply the traditional antiandrogenic drug bicalutamide after the new anti-androgen medication (i.e., AA) was administrated in an early stage, whereas sequential usage of docetaxel was still valid. It is noticeable, however, that though AA has been officially certified as the Medicare drugs for mCRPC treatment in China since 2018, most of the newly diagnosed mHSPC patients in China still cannot use AA in the early stage due to the huge cost and some other limitations. Admittedly, the second-line antiandrogenic medications are also beneficial to PCa patients. Iversen et al. conducted a three complementary, double-blind, placebocontrolled trial and found that bicalutamide, either as monotherapy or adjuvant to standard care, could significantly improve progression-free survival in patients with locally advanced PCa (22). Results from a phase III trial showed that traditional antiandrogen agent ketoconazole could postpone PCa progression and was eligible for deferred treatment with chemotherapy as well as improved the quality of life of those patients (23). In some regions of China, bicalutamide and docetaxel are still the first options of treatment for patients who cannot afford the new antiandrogenic medications.
Presently, however, the mentioned series of studies (i.e., CHAARTED, LATTITUDE, COU-AA-301, and COU-AA-302 study) have only focused on the efficacy of early or initial usage of AA, but few relevant studies explored the treatment outcomes of secondary usage of AA in advanced PCa. In this study, we observed that the patient with $\mathrm{mCRPC}$ gained treatment benefits from the retreatment of AA after the initial usage of AA 27 months ago. We hypothesized that for the observation that AA was still effective after an interval of 3 years could be related to the following issues. We should know that the proportion of PCa cells resisted to AA has been increased gradually, resulting in the disease progressed from mHSPC to mCRPC stage. After the initial withdrawal of AA, the remained sensitive cells continued to proliferate, which contributed to the validity and reliability of the retreated of AA after a specified interval. This observation suggesting that the retreatment of AA for men with mCRPC may be a novel therapeutic strategy for extending the efficiency of AA. Nevertheless, it is worth emphasizing that maybe not all of the patients with mCRPC gain treatment benefit from secondary usage of AA due to the existence of the drug-resistant by monotherapy or between multiple drugs. A critical point is to make an overall consideration and rational judgment of the withdrawal time of the initial AA treatment. Most of the current studies focused on when to start the treatment of AA but lack of withdrawal criteria. Based on this study, we speculate that the early withdrawal of AA in a proper time may be beneficial for the mCRPC patients thus to improve the efficacy of secondary usage of AA, which may present a new researching direction about this issue in the future study.

For the present case, the therapeutic value of RP in the treatment of oligometastatic PCa is also worth further discussion. Hellman and Weichselbaum (24) jointly introduced the concept of "oligometastases" in 1995. Oligometastases is a period of moderate biological invasiveness of tumor and a transitional stage between localized disease and extensive metastasis. And the number of clinically detectable metastases is limited and the metastatic organs are specific, they do not have the genetic tendency to metastasize throughout the body. Weichselbaum and Hellman defined the number of oligometastatic lesions as less than five. Patients with oligometastatic $\mathrm{PCa}$ can achieve a prolongation of survival by specific treatment of the primary lesion (25). The treatments of oligometastatic CRPC include localized 
treatment of the primary tumor and the metastatic lesions as well as systemic treatment. Optimal cytoreductive surgery for the primary tumor lesions includes RP $(26,27)$ and transurethral resection of the prostate (TURP) (28). Previous studies have validated the therapeutic effects of radical excision and the radiotherapy of the primary lesions in treating $\mathrm{PCa}$ including oligometastatic CRPC. PCa patients under these localized treatments for the primary lesions can improve the local control rate of the tumor and gain overall survival benefits. Specific to the course of treatments of this patient, the RP surgery was successfully performed as the primary lesion of PCa was totally resected and there was no remnant of tumor tissues. Postoperative, however, the patient showed a rapid tumor progression as detected by PSA examination and bone scintigraphy. From the perspective of oncology, this patient did not obtain a short-term benefit from RP, and the long-term potential effect is unknown.

At present, there are several ongoing randomized controlled trials for the surgical treatment of oligometastatic $\mathrm{PCa}$, both of which have set tumor-specific survival as the primary outcome, but the research results will take years to be published. On the other hand, the identification of metastatic and non-metastatic $\mathrm{PCa}$, oligometastatic and extensive metastatic $\mathrm{PCa}$ is a key point when assessing the value of RP in the treatment of oligometastatic CRPC. It is still controversial over the location and number of oligometastases in the 2017 St. Gallen advanced PCa consensus conference (APCCC), so there is no consensus on the definition of oligometastases. In addition, it is not possible to fully identify the exact metastatic status of $\mathrm{PCa}$ due to a lack of accurate detection methods (29). Recent studies have shown that prostatic specific membrane antigen (PSMA) PET/CT has a higher sensitivity as compared to the bone scintigraphy examination in the recognition of mPCa (30,31). Meanwhile, PSMA PET/CT also has a promising prospect in distinguishing oligometastasis from extensive metastasis, which may challenge the current treatment strategy for mPCa. However, it is not clear whether the survival of patients can be prolonged. At present, this examination has not been widely carried out in China, and the 2019 edition of EAU guidelines (32) is only recommended for evaluating biochemical recurrence after radiotherapy or RP. Clinically, the bone scintigraphy is still the main tool for $M$ staging (14). In the extensive metastatic CRPC staging, the PCa cells can exhibit a more aggressive property. Nevertheless, it is hard to accurately judge the metastatic status belongs to "high risk" or "low risk".

In the present case, this patient is likely to underestimate the malignancy of the tumor staging before RP, and thus resulting in a poor surgical result. In the 2017 LATTITUDE study, it was suggested that Gleason scores $\geq 8$, advanced $\mathrm{T}$ staging, and bone metastases were considered to be "high tumor burden". The present patient not only met these criteria but also under such a condition that at a comparatively young age (63 years old) in the first visit, high baseline PSA level, and 0 score for performance status, thus this patient might belong to "high tumor burden" of PCa and presented with potential micrometastasis already. Accordingly, the selection of surgical indications for this patient might be an incorrect intervention. More importantly, though the stage of oligometastases can be accurately identified in $\mathrm{mPCa}$ patients, there is no high-quality RCT evidence to confirm the beneficial effect played by the primary lesion treatment. At the 2017 APCCC meeting, less than 1/3 of experts agreed with RP on the treatment of oligometastases (29). Therefore, we highlighted that the current implementation of RP in patients with "oligometastatic CRPC" should be more cautious.

Based on this retrospective analysis in a $\mathrm{mPCa}$ patient with 63 months of diagnosis and treatment course, we observed that mHSPC patient who early received AA as the initial regimen and subsequently developed in mCRPC staging has gained treatment benefit from secondary administration of AA at an interval of 27 months. This "sandwich method" with AA administered initially, withdrawal, and following some else therapies (i.e., chemotherapy, RP, and other treatments) may prolong the response time of AA retreatment, delay the progression of mCRPC, and maybe beneficial to prolong survival. The withdrawal timing for firstly AA treatment and when to re-administrate AA require further study in the future. It should be cautious about the treatment of oligometastatic CRPC with RP until the survival data from relevant RCT have been published. The next phase of the sequential treatment for this patient is still under evaluation.

\section{Conclusions}

Collectively, the present case revealed that patient with metastatic PCa received a "sandwich method" based on twice administration of AA could minor progression and gain a long-time survival. This treatment strategy may represent a potentially effective regimen for mCRPC and waiting for more clinicians to validate it. 


\section{Acknowledgments}

Funding: This work was supported by the grants from Social Development Project for the Application of Commonweal Technology (No. LGF19H050004) of Zhejiang Province.

\section{Footnote}

Reporting Checklist: The authors have completed the CARE reporting checklist. Available at http://dx.doi.org/10.21037/ tau-19-851

Conflicts of Interest: All authors have completed the ICMJE uniform disclosure form (available at http://dx.doi. org/10.21037/tau-19-851). The authors have no conflicts of interest to declare.

Ethical Statement: The authors are accountable for all aspects of the work in ensuring that questions related to the accuracy or integrity of any part of the work are appropriately investigated and resolved. This study was approved by the Human Study Ethics Committees of the Taizhou Central Hospital (Taizhou University Hospital). The patient has signature informed consent.

Open Access Statement: This is an Open Access article distributed in accordance with the Creative Commons Attribution-NonCommercial-NoDerivs 4.0 International License (CC BY-NC-ND 4.0), which permits the noncommercial replication and distribution of the article with the strict proviso that no changes or edits are made and the original work is properly cited (including links to both the formal publication through the relevant DOI and the license). See: https://creativecommons.org/licenses/by-nc-nd/4.0/.

\section{References}

1. Bray F, Ferlay J, Soerjomataram I, et al. Global cancer statistics 2018: GLOBOCAN estimates of incidence and mortality worldwide for 36 cancers in 185 countries. CA Cancer J Clin 2018;68:394-424.

2. Chen $W$, Zheng $\mathrm{R}$, Baade $\mathrm{PD}$, et al. Cancer statistics in China, 2015. CA Cancer J Clin 2016;66:115-32.

3. Aytes A, Giacobbe A, Mitrofanova A, et al. NSD2 is a conserved driver of metastatic prostate cancer progression. Nat Commun 2018;9:5201.

4. Schymura MJ, Sun L, Percy-Laurry A. Prostate cancer collaborative stage data items--their definitions, quality, usage, and clinical implications: a review of SEER data for 2004-2010. Cancer 2014;120 Suppl 23:3758-70.

5. Chinese Anticancer Association Genitourinary Oncology Committee. Chinese experts consensus on the treatment of metastatic prostate cancer 2018 edition. Zhonghua Wai Ke Za Zhi 2018,56:646-52.

6. Hellerstedt BA, Pienta KJ. The current state of hormonal therapy for prostate cancer. CA Cancer J Clin 2002;52:154-79.

7. Löffeler S, Weedon-Fekjaer H, Wang-Hansen MS, et al. "Natural course" of disease in patients with metastatic castrate-resistant prostate cancer: Survival and prognostic factors without life-prolonging treatment. Scand J Urol 2015;49:440-5.

8. Smith M, Parker C, Saad F, et al. Addition of radium-223 to abiraterone acetate and prednisone or prednisolone in patients with castration-resistant prostate cancer and bone metastases (ERA 223): a randomised, doubleblind, placebo-controlled, phase 3 trial. Lancet Oncol 2019;20:408-19.

9. Barrie SE, Potter GA, Goddard PM, et al. Pharmacology of novel steroidal inhibitors of cytochrome P450(17) alpha (17 alpha-hydroxylase/C17-20 lyase). J Steroid Biochem Mol Biol 1994;50:267-73.

10. Attard G, Reid AH, A'Hern R, et al. Selective inhibition of CYP17 with abiraterone acetate is highly active in the treatment of castration-resistant prostate cancer. J Clin Oncol 2009;27:3742-8.

11. Sweeney CJ, Chen YH, Carducci M, et al. Chemohormonal Therapy in Metastatic Hormone-Sensitive Prostate Cancer. N Engl J Med 2015;373:737-46.

12. James ND, Sydes MR, Clarke NW, et al. Addition of docetaxel, zoledronic acid, or both to first-line longterm hormone therapy in prostate cancer (STAMPEDE): survival results from an adaptive, muhiarm, multistage, platform randomised controlled trial. Lancet, 2016,387:1163-77.

13. Fizazi K, Tran N, Fein L, et al. Abiraterone plus prednisone in metastatic, castration-sensitive prostate cancer Abiraterone plus prednisone in metastatic, castration-sensitive prostate cancer. N Engl J Med 2017;377:352-60.

14. Lam TBL, MacLennan S, Willemse PM, et al. EAUEANM-ESTRO-ESUR-SIOG Prostate Cancer Guideline Panel Consensus Statements for Deferred Treatment with Curative Intent for Localised Prostate Cancer from an International Collaborative Study (DETECTIVE Study). Eur Urol 2019;76:790-813. 
15. Lowrance WT, Murad MH, Oh WK, et al. Castrationresistant prostate cancer: AUA guideline amendment 2018. J Urol 2018;200:1264-72.

16. Hoyle AP, Ali A, James ND, et al. Abiraterone in "High" and "Low-risk" Metastatic Hormone-sensitive Prostate Cancer. Eur Urol 2019;76:719-28.

17. Ryan CJ, Smith MR, Fizazi K, et al. Abiraterone acetate plus prednisone versus placebo plus prednisone in chemotherapy-naive men with metastatic castrationresistant prostate cancer (COU-AA-302): final overall survival analysis of a randomised, double-blind, placebocontrolled phase 3 study. Lancet Oncol 2015;16:152-60.

18. Fizazi K, Scher HI, Molina A, et al. Abiraterone acetate for treatment of metastatic castration-resistant prostate cancer: final overall survival analysis of the COU-AA-301 randomised, double-blind, placebo-controlled phase 3 study. Lancet Oncol 2012;13:983-92.

19. van Soest RJ, van Royen ME, de Morrée ES, et al. Crossresistance between taxanes and new hormonal agents abiraterone and enzalutamide may affect drug sequence choices in metastatic castration-resistant prostate cancer. Eur J Cancer 2013,49:3821-30.

20. Courtney KD, Taplin M. The evolving paradigm of second-line hormonal therapy options for castrationresistant prostate cancer. Curr Opin Oncol 2012;24:272-7.

21. Kao SC, Hovey E, Marx G. Second-line therapy for castrate-resistant prostate cancer: A literature review. Asia Pac J Clin Oncol 2011;7:212-23.

22. Iversen P, McLeod DG, See WA, et al. Antiandrogen monotherapy in patients with localized or locally advanced prostate cancer: final results from the bicalutamide Early Prostate Cancer programme at a median follow-up of 9.7 years. BJU Int 2010;105:1074-81.

23. Small EJ, Halabi S, Dawson NA, et al. Antiandrogen withdrawal alone or in combination with ketoconazole in androgen-independent prostate cancer patients: a phase III trial (CALGB 9583). J Clin Oncol 2004;22:1025-33.

24. Hellman S, Weichselbaum RR. Oligometastases. J Clin Oncol 1995;13:8-10.

25. Weichselbaum RR, Hellman S. Oligometastases revisited. Nat Rev Clin Oncol 2011;8:378-82.

26. Ward JF, Slezak JM, Blute ML, et al. Radical prostatectomy for clinically advanced (cT3) prostate cancer since the advent of prostate-specific antigen testing: 15year outcome. BJU Int 2005;95:751-6.

27. Johnstone PA, Ward KC, Goodman M, et al. Radical prostatectomy for clinical $\mathrm{T} 4$ prostate cancer. Cancer 2006;106:2603-9.

28. Qin XJ, Ma C, Ye D, et al. Tumor cytoreduction results in better response to androgen ablation-a preliminary report of palliative transurethral resection of the prostate in metastatic hormone sensitive prostate cancer. Urol Oncol 2012;30:145-9.

29. Gillessen S, Attard G, Beer M, et al. Management of Patients with Advanced Prostate Cancer: The Report of the Advanced Prostate Cancer Consensus Conference APCCC 2017. Eur Urol 2018;73:178-211.

30. Rauscher I, Düwel C, Haller B, et al. Efficacy, Predictive Factors, and Prediction Nomograms for Ga-labeled Prostate-specific Membrane Antigen-ligand Positronemission Tomography/Computed Tomography in Early Biochemical Recurrent Prostate Cancer After Radical Prostatectomy. Eur Urol 2018;73:656-61.

31. Budäus L, Leyh-Bannurah SR, Salomon G, et al. Initial Experience of (68)Ga-PSMA PET/CT Imaging in High-risk Prostate Cancer Patients Prior to Radical Prostatectomy. Eur Urol 2016;69:393-6.

32. Mottet N, van den Bergh RCN, Briers E, et al. EAU ESTRO - ESUR - SIOG Guidelines on Prostate Cancer. European Association of Urology, 2019. Available online: https://uroweb.org/guideline/prostate-cancer/
Cite this article as: Wang H, Li X, Zhang P, Liu S, Xie Q, Zhao S. Treatment gains from the "sandwich method" of abiraterone acetate for men with metastatic prostate cancer: a case report and sharing of our experience. Transl Androl Urol 2020;9(3):1448-1458. doi:10.21037/tau-19-851 


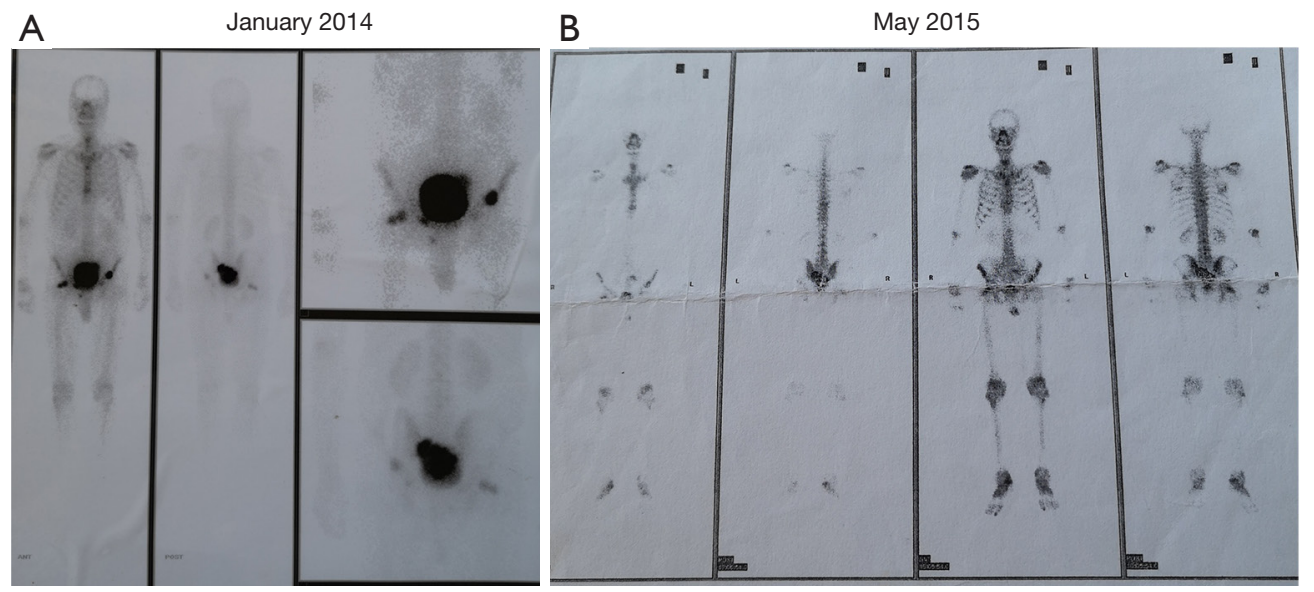

Figure S1 Bone scintigraphy findings in other hospitals. (A) Initial evaluation in January 2014; (B) assessment at the end of the first course of treatment of AA in May 2015. AA, abiraterone acetate. 